\title{
Vólvulo de ciego
}

\section{Cecal volvulus}

\author{
Fernando Revoredo-Rego ${ }^{1, a}$, Juan Luna-Cydejko ${ }^{1, b}$, Gustavo Reaño-Paredes ${ }^{1, c}$, Fritz Kometter-Barrios ${ }^{1, d}$, Yuri López-Zenteno ${ }^{2, e}$, \\ Carlos Wong-Chu ${ }^{1, \mathrm{f}}$ \\ ${ }^{1}$ Servicio de Cirugía General, Clínica Internacional. Lima, Perú.G \\ ${ }^{2}$ Servicio de Radiología, Clínica Internacional. Lima, Perú. \\ ${ }^{a}$ Médico cirujano general, ORCID: https://orcid.org/0000-0002-8316-9703 \\ ${ }^{\mathrm{b}}$ Médico cirujano general, ORCID: https://orcid. org/0000-0002-0826-6589 \\ 'Médico cirujano general, ORCID: https://orcid. org/0000-0002-4298-631x \\ ${ }^{d}$ Médico cirujano general, ORCID: https://orcid.org/0000-0002-2930-8362 \\ ${ }^{\text {e }}$ Médico radiólogo, ORCID: https://orcid. org/0000-0003-3596-2050 \\ ${ }^{\dagger}$ Médico cirujano general
}

An Fac med. 2021;82(3):225-8. / DOI: https://doi.org/10.15381/anales.v82i3.17604

\section{Correspondencia:}

Fernando Revoredo Rego

fernandorevoredo@hotmail.com

Recibido: 17 de diciembre 2020

Aprobado: 19 de septiembre 2021

Publicación en línea: 28 de septiembre 2021

Conflictos de interés: Los autores declaran no tener conflictos de interés.

Fuente de financiamiento: Los autores declaran no tener conflictos de interés.

Autofinanciado: Autofinanciado

Citar como: Revoredo-Rego F, LunaCydejko J, Reaño-Paredes G, KometterBarrios F, López-Zenteno Y, Wong-Chu C. Vólvulo de ciego. An Fac med. 2021;82(3):225-8. DOI: https://doi. org/10.15381/anales.v82i3.17604

\section{Resumen}

El vólvulo de ciego involucra al colon derecho proximal, el ciego y el ileon terminal. Se necesitan dos requisitos, una fijación incompleta del ciego y la limitación del movimiento intestinal en un punto fijo que sirve como apoyo y eje para la rotación. Presentamos el caso de una mujer de 52 años que presentó una obstrucción intestinal aguda. Se hizo el diagnóstico preoperatorio con una tomografía y se realizó una colectomía derecha asistida por laparoscopia con anastomosis primaria. En estos pacientes se recomienda la colectomía derecha no oncológica ya que elimina por completo la posibilidad de recurrencia. No se recomienda la colonoscopía, la cecopexia, ni la cecostomía por su alto riesgo de recurrencia y morbimortalidad asociadas.

Palabras clave: Vólvulo Intestinal; Ciego; Obstrucción Intestinal (fuente: DeCS BIREME).

\section{Abstract}

Cecal volvulus involves the proximal right colon, the cecum and the terminal ileum. Two requirements are needed, an incomplete fixation of the cecum and the restriction of the bowel movement at a fixed point which serves as a fulcrum for rotation. We present a case of a 52-year-old woman who was admitted with the diagnosis of an acute intestinal obstruction. The preoperative diagnosis was made with a tomography and she underwent a laparoscopic assisted right colectomy with primary anastomosis. In these patients, the right colon nononcologic surgical resection is recommended because eliminates the possibility of volvulus recurrence. Colonoscopy, cecopexy, or cecostomy should be avoided because its associated significant recurrence, morbidity and mortality.

Keywords: Intestinal Volvulus; Cecum; Intestinal Obstruction (source: MeSH NLM). 


\section{INTRODUCCIÓN}

El vólvulo de colon consiste en la torsión de un segmento del intestino alrededor de su propio mesenterio ${ }^{(1,2,3)}$. Esta patología representa la tercera causa de obstrucción colónica en el mundo, después del cáncer colorrectal y la diverticulitis sigmoidea complicada ${ }^{(1,2)}$. La incidencia del vólvulo de colon varia en las diferentes regiones del mundo, existiendo un área endémica conocida como "el cinturón del vólvulo" (África, América del Sur, Rusia, Europa del Este, el Medio Este e India), donde el vólvulo representa hasta el $42 \%$ de todas las obstrucciones intestinales ${ }^{(2)}$, mientras que, en países occidentales (norte América, Europa Occidental, Australia), el vólvulo de colon representa menos del $5 \%$ de las obstrucciones intestinales ${ }^{(1,2)}$. Se ha postulado que esta diferencia es causada por diferencias anatómicas y diferencias en la dieta, altitud, factores culturales e infecciones endémicas ${ }^{(1)}$.

El vólvulo de colon se presenta más comúnmente en el colon sigmoides $(60 \%$ - 75\%), seguido del ciego (25\% - 40\%), y raramente el colon transverso (1\% - 4\%) y la flexura esplénica (1\%) ${ }^{(1)}$. El vólvulo de ciego anatómicamente involucra al íleon terminal, ciego y colon derecho proximal ${ }^{(4)}$. Se han descrito dos variantes de vólvulo cecal, la primera consiste en la rotación axial del colon derecho proximal, el ciego y el íleon terminal alrededor de su mesenterio ${ }^{(4,5)}$. Esta variante representa un $90 \%$ de los casos de vólvulo de ciego ${ }^{(2)}$ y generalmente da como resultado obstrucción intestinal, isquemia, necrosis y peritonitis fecal ${ }^{(5)}$. La otra variante consiste en el plegamiento anterosuperior del ciego sin rotación axial, más comúnmente conocida como "báscula cecal" $(10 \%)^{(2,6,7)}$ y causa menos compromiso vascular porque no hay una verdadera torsión mesentérica ${ }^{(1,4)}$.

La incidencia del vólvulo cecal es de 2,8 a 7,1 por millón de personas por año, y representa del $1 \%$ a $1,5 \%$ de todas las obstrucciones intestinales en adultos $(4,6,7,8)$. La etiología de esta patología está relacionada con la embriogénesis tardía, en la que el ciego rota en sentido anti horario desde el lado izquierdo del abdomen al cuadrante inferior derecho ${ }^{(2,4)}$. A la vez que esto ocurre, el mesenterio del colon derecho se fija a las estructuras retroperitoneales ${ }^{(4,5,6)}$; si la fijación es incompleta, el ciego queda móvil, uno de los requisitos para el vólvulo de ciego ${ }^{(1,5,7)}$. El otro requisito es la limitación del movimiento intestinal en un punto fijo (adherencias post operatorias, obstrucción parcial o desplazamiento por una masa abdominal), que sirven de puntos de apoyo (fulcros) y ejes para la rotación del ciego móvil ${ }^{(5,6,9)}$.

Nuestro objetivo es presentar un caso de vólvulo de ciego y hacer una revisión actualizada, en lo referente a la presentación clínica, diagnóstico y manejo de los pacientes con esta patología.

\section{REPORTE DE CASO}

Paciente mujer de 52 años, ingresó a emergencia con distensión y dolor abdo- minal de tipo cólico, intenso, localizado en hemiabdomen derecho; de 24 horas de evolución. Como antecedentes, la paciente había tenido una colecistectomía laparoscópica 8 años antes y una histerectomía 6 años antes. En el examen físico, los signos vitales estables, y a nivel abdominal, había una distensión marcada del hemiabdomen derecho, ruidos hidroaéreos aumentados y dolor difuso a la palpación. Los exámenes de laboratorio de rutina estuvieron dentro de límites normales. La tomografía (Figura 1) informó una gran dilatación del colon derecho (hasta 7,5 cm) y nivel hidroaéreo, ciego en hipocondrio derecho, y hallazgos asociados a "signo del remolino" y liquido libre en el espacio parietocólico derecho y cavidad pélvica, así como edema de pared del extremo distal del íleon.

Con el diagnóstico de vólvulo de ciego, se programó para una laparoscopía de emergencia. Los hallazgos fueron: bridas en región íleocecal, colon derecho móvil, con meso redundante, rotado $180^{\circ}$ en sentido horario, edema marcado de asas delgadas y gruesas, líquido libre de aspecto inflamatorio en abdomen (Figuras 2 y 3). Se realizó una colectomía derecha no oncológica y se amplió el puerto umbilical para extraer la pieza operatoria y se hizo la anastomosis íleo-transversa latero-lateral con auto sutura gastrointestinal $\left(G I A^{\circledR}\right)$. La paciente inició dieta en el tercer día del post operatorio y fue dada de alta en el quinto día del post operatorio sin complicaciones. Los controles post operatorios fueron sin incidencias y las heridas de los puertos de trabajo cicatrizaron adecuadamente.

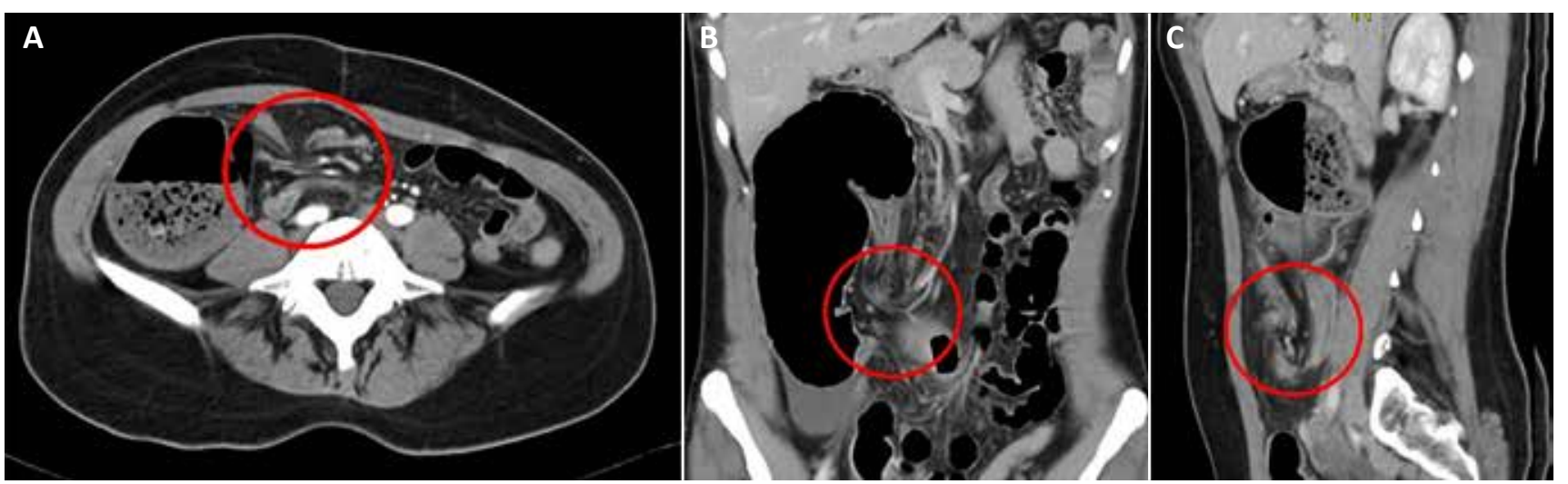

Figura 1. A. Imagen de tomografía que muestra el "signo del remolino". Nótese también la marcada distensión del hemiabdomen derecho. B y C. Muestran también el "signo del remolino" 

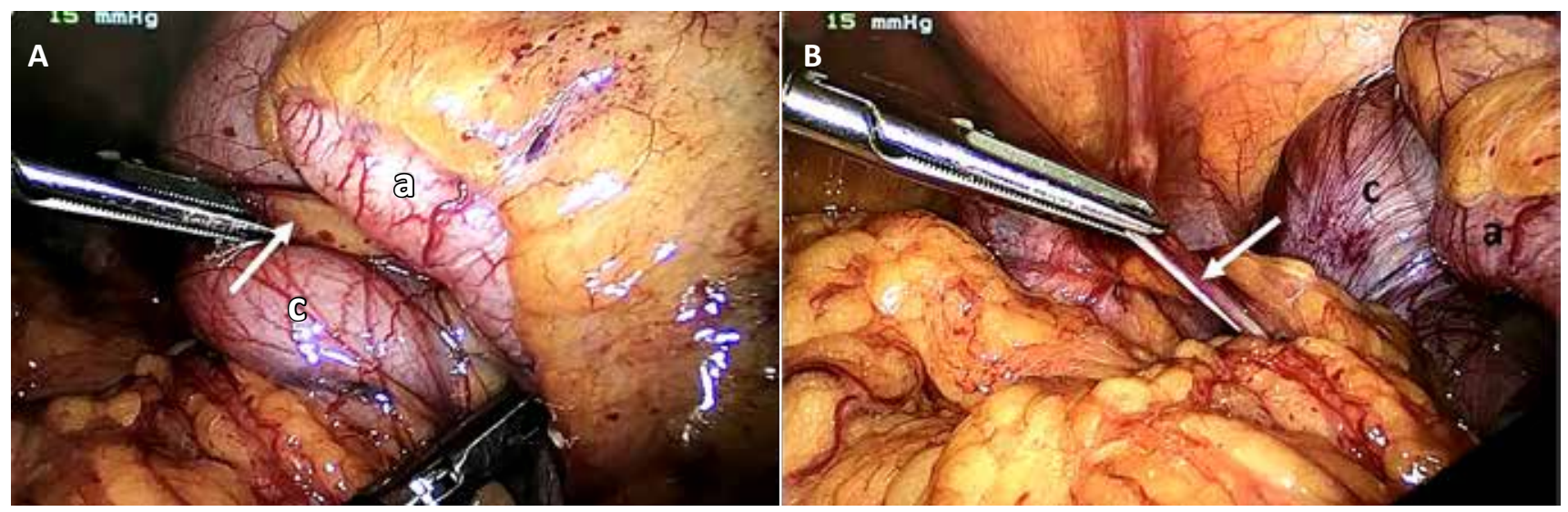

Figura 2. A y B. Muestran los hallazgos de la laparoscopía. La flecha blanca muestra la brida, alrededor de la cual la región íleo cecal esta volvulada. (a: apéndice cecal, c: ciego)

El estudio anatomo-patológico informó una pared colónica adelgazada, mucosa con marcada congestión vascular. La lámina propia con moderado infiltrado inflamatorio. La capa muscular adelgazada y la serosa con vasos congestivos. El apéndice cecal con congestión vascular e hiperplasia folicular reactiva.

Actualmente la paciente tiene 27 meses de seguimiento asintomática.

\section{DISCUSIÓN}

El $73 \%$ de los pacientes con vólvulo de ciego son mujeres y el $27 \%$ son varones ${ }^{(9)}$, con un pico de incidencia a los 50 años ${ }^{(3)}$, muchas de ellas con algún tipo de cirugía pélvica (histerectomías), que generan adherencias post operatorias y que podrían formar un eje alrededor del cual el ciego puede rotar en personas anatómicamente predispuestas (fijación incompleta del mesenterio del colon derecho) ${ }^{(3,9)}$. Además de la cirugía abdominal previa (23\% a 53\% de pacientes) ${ }^{(5,6,7)}$, se han descrito otros factores asociados al vólvulo de ciego como el embarazo, ingesta alta de fibra, íleo adinámico, estreñimiento crónico y la obstrucción colónica distal ${ }^{(7,9,10,11)}$. La paciente que reportamos tenía 52 años, con antecedente de histerectomía previa y con un colon derecho móvil, es decir una paciente anatómicamente predispuesta y con adherencias por la cirugía pélvica previa.

La presentación clínica del vólvulo de ciego es inespecífica ${ }^{(2,10,12)}$. Puede haber episodios intermitentes de distensión abdominal, cólico abdominal, estreñimiento, náuseas y vómitos que se pueden resolver espontáneamente y luego recurren; cuadro conocido como "síndrome del ciego móvil” $(2,4,6,7)$. En otros casos, el vólvulo se presenta como una obstrucción intestinal aguda ${ }^{(1,2)}$, caracterizada por cólicos abdominales y vómitos que no se resuelven espontáneamente. En el examen físico se puede encontrar dolor abdominal con o sin masa palpable y ruidos hidroaéreos aumentados. Si estos pacientes no son tratados pueden desarrollar estrangulación y perforación intestinal ${ }^{(1,2,6,8)}$. La tercera forma de presentación clínica descrita es la obstrucción fulminante, caracterizada por dolor abdominal intenso, asociado a shock séptico, peritonitis y necrosis intestinal ${ }^{(1,4,6)}$. El cuadro clínico de nuestra paciente fue de obstrucción intestinal aguda, por lo que fue programada para una laparoscopía de emergencia.

Los estudios de laboratorio no son específicos para el diagnóstico de vólvulo
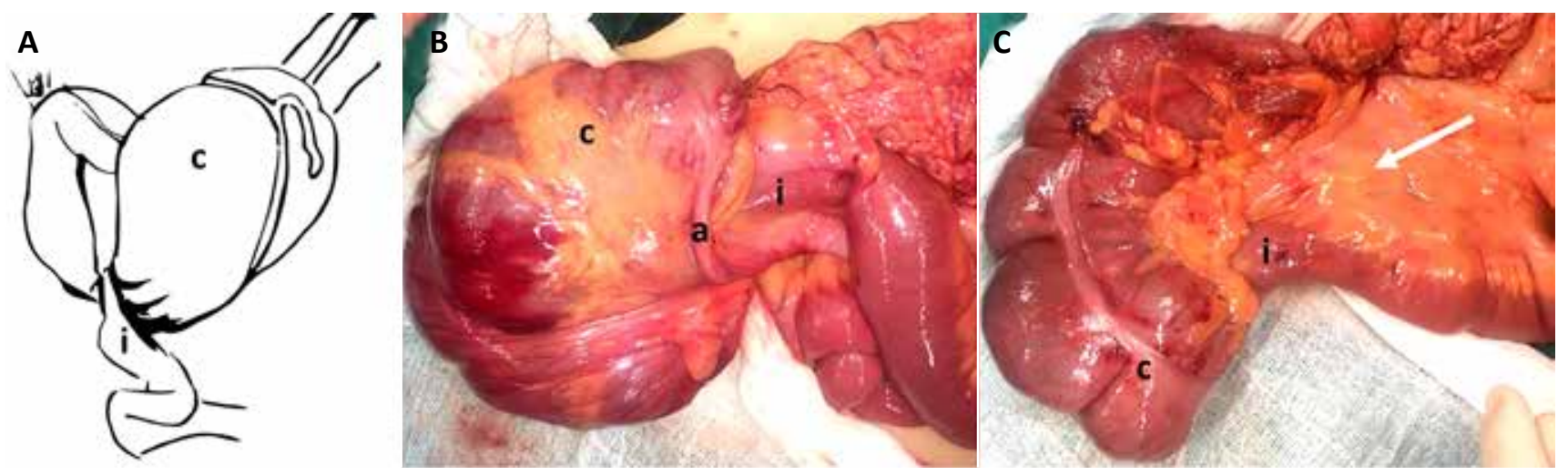

Figura 3. A. Esquema de la rotación horaria de la región íleo cecal. B. Vólvulo de la región íleo cecal, en sentido horario. C. Disposición normal de la región íleo cecal. (a: apéndice cecal, c: ciego, i: íleon) 
de ciego ${ }^{(2)}$ y están determinados por el patrón, la severidad y la duración de la obstrucción intestinal ${ }^{(6)}$. La tomografía abdominal multicorte es el método radiológico más preciso para el diagnóstico del vólvulo de ciego ${ }^{(1,7)}$, con una sensibilidad de $100 \%$ y una especificidad de $90 \%{ }^{(1,2,8)}$. Puede detectar signos de gravedad, estos incluyen signos directos de isquemia intestinal como la neumatosis intestinal (isquemia arterial) y engrosamiento de la pared intestinal (isquemia venosa). Los signos indirectos de isquemia intestinal son la embolia gaseosa portal o mesentérica y neumoperitoneo sugerente de perforación ${ }^{(1,2)}$. Una ventaja adicional de la tomografía es que nos ayuda a descartar otras posibilidades del diagnóstico diferencial, particularmente el cáncer colorrectal obstruido ${ }^{(2)}$.

Los signos tomográficos descritos son la dilatación marcada del ciego, "el grano de café", caracterizado por un ciego dilatado, lleno de aire y líquido, en una visión axial ${ }^{(2,6)}$. El "signo del pico de pájaro", caracterizado por el estrechamiento progresivo del extremo intestinal aferente y eferente que termina en el sitio de torsión ${ }^{(1,6)}$. El "signo del remolino", que consiste en una imagen que contiene hebras de tejido blando y grasa arremolinadas en el punto de torsión alrededor del cual las asas intestinales y los vasos mesentéricos giran ${ }^{(1,2,10)}$. En el caso de nuestra paciente los hallazgos tomográficos incluyeron una dilatación marcada del colon derecho y el "signo del remolino" asociado a liquido libre en el espacio parietocólico derecho y cavidad pélvica. No se encontraron signos radiológicos directos o indirectos de isquemia intestinal.

Debido a lo inusual de esta patología, no existen estudios prospectivos que guíen las decisiones de tratamiento ${ }^{(6)}$. El vólvulo de ciego se debe considerar como una emergencia quirúrgica, aún en los casos de ausencia clínica o radiológica de gravedad $^{(1,2,11)}$, debido a que la pared delgada del ciego es especialmente sensible a la dilatación, isquemia y perforación ${ }^{(3)}$. Aproximadamente $61 \%$ de los vólvulos de ciego se necrosan ${ }^{(1)}$, por esta razón no se recomienda el manejo conservador ${ }^{(12)}$. La necrosis colónica y la peritonitis son los dos factores que incrementan el riesgo de mortalidad ${ }^{(1,2,3)}$. Actualmente, la recomendación para el manejo del vólvulo de ciego es la hemicolectomía derecha no oncológica ${ }^{(1,2,9,11)}$, con anastomosis primaria $u$ ostomía; esto dependerá de las condiciones locales (contaminación) y el estado hemodinámico del paciente ${ }^{(1,5,10)}$. Cuando se decida hacer anastomosis primaria, se recomienda que sea latero - lateral ${ }^{(1,2)}$, por la disparidad de los lúmenes del íleon y el colon derecho ${ }^{(1)}$. La resección intestinal elimina por completo la posibilidad de recurrencia ${ }^{(6,8,10,14)}$ con baja morbilidad ${ }^{(12)}$. La paciente que reportamos era joven, hemodinámicamente estable y no tenía perforación ni contaminación fecal, por lo que se realizó una anastomosis primaria luego de la resección.

Debe evitarse la colonoscopía en el vólvulo cecal debido a su baja eficacia $(30 \%)^{(1,2,6,8)}$, el alto riesgo de recurrencia (hasta $70 \%)^{(3)}$, el potencial riesgo de perforación colónica y el retraso en el tratamiento quirúrgico ${ }^{(6,8)}$.

Además de la resección intestinal, se han descrito otras opciones quirúrgicas, que incluyen la reducción manual sola con una morbilidad de $25 \%$ y una recurrencia de hasta $70 \%{ }^{(3,6,8)}$. La cecopexia, con una mortalidad de $30 \%$ y una recurrencia de hasta $40 \%{ }^{(3,6,7)}$. La colocación de un tubo de cecostomía con una morbilidad de $52 \%{ }^{(4)}$, mortalidad de $40 \%$ y una recurrencia de $33 \%{ }^{(6,7)}$. Ninguno de estos procedimientos se recomienda debido a sus altas tasas de morbilidad y recurrencia ${ }^{(2,9)}$.

El abordaje quirúrgico tradicional de esta patología fue la cirugía abierta; sin embargo, actualmente hay reportes del uso de la laparoscopía para el tratamiento estos pacientes ${ }^{(1,7,8,9)}$, siendo aproximadamente el $3 \%$ de casos reportados los que fueron abordados por laparoscopía (9). Se recomienda realizar un neumoperitoneo abierto por el riesgo de lesionar el colon dilatado ${ }^{(13)}$. Otra recomendación es que se puede ampliar el puerto umbilical para la extracción del ciego previamente descomprimido ${ }^{(3,8)}$ y la realización de la resección y anastomosis ${ }^{(13)}$. En el caso que presentamos, se trataba de una paciente sin comorbilidades, hemodiná- micamente estable y sin signos radiológicos de isquemia intestinal, por lo que se optó por un abordaje laparoscópico para la resección intestinal no oncológica y la anastomosis primaria.

Se puede concluir que el vólvulo de ciego es una patología rara, con una presentación clínica inespecífica y que debe considerarse como una emergencia quirúrgica, siendo la laparoscopía una opción segura y efectiva en pacientes seleccionados.

\section{REFERENCIAS BIBLIOGRÁFICAS}

1. Bauman ZM, Evans $\mathrm{CH}$. Volvulus. Surg Clin N Am. 2018; 98(5): 973 - 993. DOI: 10.1016/j. suc.2018.06.005.

2. Perrot L, Fohlen A, Alves A, Lubrano J. Management of the colonic volvulus in 2016. J Visc Surg. 2016; 153(3): 183 - 192. DOI: 10.1016/j.jviscsurg.2016.03.006

3. Halabi WJ, Jafari MD, Kang CY, Nguyen VQ, Carmichael JC, Mills S, et al. Colonic volvulus in the United States. Trends, outcomes, and predictors of mortality. Ann Surg. 2014; 259(2): 293 - 301. DOI: 10.1097/SLA.0b013e31828c88ac

4. Gingold D, Murrell Z. Management of colonic volvulus. Clin Colon Rectal Surg. 2012; 25 (4): 236 - 244. DOI: 10.1055/s-0032-1329535

5. Mizuguchi Y, Mamada Y, Shimizu T, Kanda T, Furk $\mathrm{H}$, Akama $\mathrm{Y}$, et al. Cecal volvulus following elective laparoscopic cholecystectomy: a case report. J Nippon Med Sch. 2016; 83(4): 172 - 176. DOI: 10.1272/jnms.83.172

6. Consorti ET, Liu TH. Diagnosis and treatment of caecal volvulus. Postgrad Med J. 2005; 81: 772 776. DOI: 10.1136/pgmj.2005.035311

7. Ramirez-Ramirez MM, Villanueva-Sáenz E, Ramírez-Wiella-Schwuchow G. Colectomía laparoscópica electiva por vólvulo cecal: reporte de un caso y revisión de la literatura. Cir Cir. 2017; 85(1): 87 - 92. DOI: 10.1016/j.circir.2016.03.003

8. Kagawa $Y$, Kato T, Naito A, Morimoto $Y$, Sato $Y$, Kuwahara $R$, et al. Single-site laparoscopic right hemicolectomy for acute cecal volvulus: a case report. Surg Case Rep. 2016; 2(1): 51. DOI: 10.1186/ s40792-016-0179-9

9. Bailey KS, Lundstrom E, Borgstrom D. Cecal volvulus: an evolving disease. Am Surg. 2018; 84(9): e418 - e419.

10. Ozgun YM. A rare cause of acute abdomen in a Young patient: cecal volvulus. Ulus Cerrahi Derg. 2015; 31: 105 - 106. DOI: 10.5152/UCD.2013.13

11. Habre J, Sautot-Vial N, Marcotte C, Benchimol D. Caecal volvulus. Am J Surg. 2008; 196(5): e48 e49. DOI: 10.1016/j.amjsurg.2007.11.029

12. Lee SY, Bhaduri M. Cecal volvulus. CMAJ. 2013; 185(8): 684. DOI: 10.1503/cmaj.120651

13. Kelly MD, Bunni J, Pullyblank A. Laparoscopic assisted right hemicolectomy for caecal volvulus. World J Emerg Surg. 2008; 3: 4. DOI: 10.1186/1749-7922-3-4 